\title{
The extraction of liquid, protein molecules and yeast cells from paper through surface acoustic wave atomization
}

Qi, Aisha; Yeo, Leslie; Friend, James; Ho, Jenny

https://researchrepository.rmit.edu.au/esploro/outputs/9921858401401341/filesAndLinks?institution=61RMIT_INST\&index=null

Qi, A., Yeo, L., Friend, J., \& Ho, J. (2010). The extraction of liquid, protein molecules and yeast cells from paper through surface acoustic wave atomization. Lab On a Chip: Miniaturisation for Chemistry, Physics, Biology and Bioengineering, 10(4), 470-476. https://doi.org/10.1039/b915833b

Document Version: Accepted Manuscript

Published Version: https://doi.org/10.1039/b915833b

Repository homepage: https://researchrepository.rmit.edu.au

(C) 2010 The Royal Society of Chemistry

Downloaded On 2023/04/27 01:31:36 +1000 


\title{
The Extraction Of Liquid, Protein Molecules and Yeast Cells From Paper Through Surface Acoustic Wave Atomization
}

\author{
Aisha Qi, Leslie Yeo, and James Friend \\ MicroNanophysics Research Laboratory, \\ Department of Mechanical and Aerospace Engineering, \\ Monash University, Melbourne VIC 3800 Australia. \\ Jenny Ho \\ Bio-Engineering Laboratory, Department of Chemical Engineering, \\ Monash University, Melbourne VIC 3800 Australia. \\ (Dated: October 12, 2009)
}

\begin{abstract}
Paper has been proposed as an inexpensive and versatile carrier for microfluidics devices with abilities well beyond simple capillary action for pregnancy tests and the like. Unlike standard microfluidics devices, extracting a fluid from the paper is a challenge and a drawback to its broader use. Here we extract fluid from narrow paper strips using surface acoustic wave (SAW) irradiation that subsequently atomizes the extracted fluid into a monodisperse aerosol for use in mass spectroscopy, medical diagnostics, and drug delivery applications. Two protein molecules, ovalbumin and bovine serum albumin (BSA) have been preserved in paper and then extracted using atomized mist through SAW excitation; protein electrophoresis shows there is less than $1 \%$ degradation of either protein molecule in this process. Finally, a solution of live yeast cells was infused into paper, which was subsequently dried for preservation then remoistened to extract the cells via SAW atomization, yielding live cells at the completion of the process.The successful preservation and extraction of fluids, proteins and yeast cells significantly expands the usefulness of paper in microfluidics.
\end{abstract}




\section{INTRODUCTION}

Paper as a fluid carrier has a long history, from the early nineteenth century and the first use of litmus paper [1] to paper chromatography [2] and even the simple home pregnancy kit introduced in the early 1970s [3]. Today, effective and affordable medical diagnostics and therapeutics are essential to improving the quality and length of life in rich and poor countries alike [4]. Inexpensive, portable, and simple microfluidic devices are perceived as a potential means to achieving this goal. In diagnosis of both communicable diseases carried by microbes, parasites and viruses, and chronic diseases like cancer, atherosclerosis and emphysema, microfluidics has been shown to be an effective option $[4,5]$, albeit with the limitations of cost in fabrication, an issue addressed with the use of paper. Recently, Whitesides, et al. [6, 7], formed hydrophobic barriers and hydrophilic microchannels in paper to create separate zones for diagnostic protocols - using, for example, different assay reagents-and providing a way to manipulate fluid inside the paper. Martinez, et al. [8], wicked a fluid sample (artificial urine) into a paper-based microfluidic diagnostic device containing glucose and protein assay reagents in separate zones. Their approach enabled inexpensive diagnosis of kidney disease and metabolic problems through the measurement of the concentration of glucose and various proteins using digital photos of reagent-treated regions in paper.

Once the fluid is in the paper it must remain there, precluding, for example, extraction and collection of the processed fluid to perform more sophisticated analysis outside the paper. If the hope is to carry out more than just simple in-paper diagnosticssay chemical reactions [9] or mass spectroscopy [10] - a method for extracting the fluid out of the paper is necessary. We describe such an approach where fluids may be extracted from the paper and atomized. In combining the flexibility and economy of the lab-in-paper approach with the ability to extract the result from the paper and use it in other ways, perhaps in more accurate and quantifiable laboratory techniques, a variety of potential applications of lab-in-paper is made possible.

Atomization, in particular, is critical to various industrial processes, including fuel injection, agricultural sprays, ink-jet printing, and many other areas; for example, mass spectrometry, DNA microarray printing, and pulmonary drug delivery [11-13]. Pointof-care pulmonary drug delivery via portable nebulization is a tremendous opportunity 
for atomizer devices should a way be found to make a nebulizer both small enough to easily carry and flexible enough to accommodate the limitations of the drugs to be delivered [14-16] without compromising patient safety [17]. There are many methods for atomization, including hydrodynamic flow focusing [18], electrospraying [12, 19], ultrasonic atomization [20] and other vibration-induced atomization methods [21]. Compared to these approaches, surface acoustic wave (SAW) atomization offers many advantages-tiny devices commensurate with microfluidics technology, millisecondorder response, lower costs, and reduced power consumption [22-24] - and for these reasons it is considered in more detail below.

SAW atomization operates at much higher frequencies (10-500 MHz) than conventional ultrasound devices (20 kHz-3 MHz), using transverse-axial polarized elliptical electro-acoustic waves traveling across the atomizer's substrate surface. Though the amplitude of a SAW is only a few nanometers, the acceleration of the surface is incredibly high $\left(10^{7} \mathrm{~m}^{2} / \mathrm{s}\right)$ because of the high operating frequency. Therefore, when a SAW is transmitted into a liquid drop placed upon the substrate, it is able to not only form capillary waves across the free surface of the drop but also drives it to break up into a mist of droplets with an average, controlled diameter of 1-10 $\mu \mathrm{m}$ [22]. Micro and nanoparticles may be formed via controlled evaporation of these droplets [25-27], but regardless of the desired product, the challenge is in maintaining a relatively stationary free fluid surface on the SAW device as an atomization source.

In the past, a sessile drop (normally $1-3 \mu$ l) has been used, but it can easily be transported across the surface [28], perhaps causing a thin film to be drawn out [29] and consequently reducing the aerosol droplet diameter [22], or even pushing the droplet entirely out of the SAW irradiation field, causing the atomization to cease. Though patterning the surface's hydrophobicity can help, the use of hydrophobic films tends to absorb the SAW, and the use of soft polymer microfluidics materials like PDMS to form channels can be problematic, though some have been quite successful in pumping fluids in PDMS microchannels using SAW [30,31], with the power we are using in atomization the PDMS tends absorb the SAW so strongly that it is easily damaged [32]. Further, if the sessile source drop is not continuously replenished, it rapidly decreases in size during atomization and changes the form of atomization once it falls below a critical size, affecting the monodispersity of the droplets. Using a supply needle or capillary 
tube as in [29] is feasible, but while the drop is in contact with this supply structure, the SAW is transmitted into the structure, strongly affecting the atomization performance. Dripping drops onto the surface combines both these problems; not only is there a nonuniform source drop size but also the periodic effect of having the needle in contact as the fluid drips. Finally, there is the issue of requiring an external pump to provide the fluid for atomization, that, due to its size and power requirements, adds to the difficulty of making a portable and economic microfluidic device.

Paper, properly used, solves all these problems. Using hydrophilic paper to siphon liquid from a supply reservoir to the surface of the SAW device provides the atomizer with continuous flow without the need for pumping; the paper will further be shown to permit atomization without affecting the performance of the SAW device, offering a means to form and constrain a fluid meniscus along the paper's edge. Though the self-pumping effect of SAW atomization was very briefly noted by Kurosawa, et al. [33], while Chono, et al. [34], considered using filter paper in a crude scheme to control atomization, we combine these concepts in a detailed study to demonstrate the potential of lab-in-paper technology to supply fluids for use outside the paper and to extract chemicals and intact cells out of paper within the extracted fluid.

\section{METHODS}

\section{A. The SAW device}

The SAW atomizer is made of a low-loss piezoelectric substrate, $127.86^{\circ} y$ - $x$-rotated single-crystal lithium niobate, with chromium-aluminum single-phase uni-directional transducer (SPUDT) electrodes patterned via UV lithography [24], as shown in Fig. 1(a)(b). Unlike traditional SAW devices patterned with interdigital transducer (IDT) electrodes, SAW generated from SPUDT devices propagates in a single direction [35], thus less energy is lost and the risk of interfering reflections is reduced. The focused design provides concentrated energy to the desired position: the focusing point.

The resonant frequency of the atomizer is determined by $f_{s}=c_{s} / \lambda_{\mathrm{SAW}}$, where $c_{s}=3965 \mathrm{~m} / \mathrm{s}$ is the speed of the SAW traveling along the substrate surface. A sinusoidal electrical signal is supplied to the substrate through the SPUDT, generating a Rayleigh 
wave SAW which propagates across the substrate surface. The wavelength $\lambda_{\text {SAW }}$ is about $132 \mu \mathrm{m}$, as stipulated by $\lambda_{\text {SAW }}=c_{s} / f_{s}$, where $f_{s}=30 \mathrm{MHz}$ is the desired frequency. When the SAW meets the leading edge of water placed on the substrate, about $33 \%$ of the transverse portion of the Rayleigh wave refracts into the water at the Rayleigh angle, defined as $\theta_{R}=\arcsin \left(c_{l} / c_{s}\right)$, where $c_{l}$ is the propagation speed of the SAW in liquid. Therefore, with the sound speeds $c_{l}=1485 \mathrm{~m} / \mathrm{s}$ (water) and $c_{s}=3965 \mathrm{~m} / \mathrm{s}$, the Rayleigh angle is about $22^{\circ}$ [36]. As shown in Fig. 1(c), the horizontal component of the SAW subsequently causes $S A W$ streaming [37] while the vertically polarized acoustic energy travels to the liquid surface, destabilizes the free surface of the liquid, forms a capillary wave along it and thus generates micron sized aerosol droplets by destabilizing the capillary wave to break up $[22,23]$.

\section{B. Extraction and atomization of liquid from paper}

By using the SAW device shown in Fig. 1(a), atomization starts using an input power beyond $1 \mathrm{~W}$ and becomes efficient as the input power exceeds $2 \mathrm{~W}$. Beyond $2 \mathrm{~W}$ of input power, however, the atomization rate scarcely increases while the heat generated grows significantly to a point where the protein molecules and cells are damaged. Therefore, in the following experiments, the input power was set to $2.5 \mathrm{~W}$, supporting efficient SAW atomization without damaging the biomolecules.

Polyester-cellulose clean room paper (Lym-Tech, Chicopee, MA USA), as shown in Fig. 2, was used in the experiments. The experiments were designed as follows:

Extraction and atomization of liquid from paper. A high-speed camera (MC1310, Mikrotron, Germany) with a long distance microscopic lens (InfiniVar, USA) was employed to view the atomization at 2754 frames per second such that the extraction of liquid and contact conditions between the paper and the SAW device can be directly observed. Then, by reducing the size of paper strip and fitting it into a capillary tube, the liquid can be automatically provided onto the SAW device from a reservoir. A red dye-laden paper strip was also employed to show that the atomized liquid was able to extract dye molecules from the paper into the aerosol mist ( 


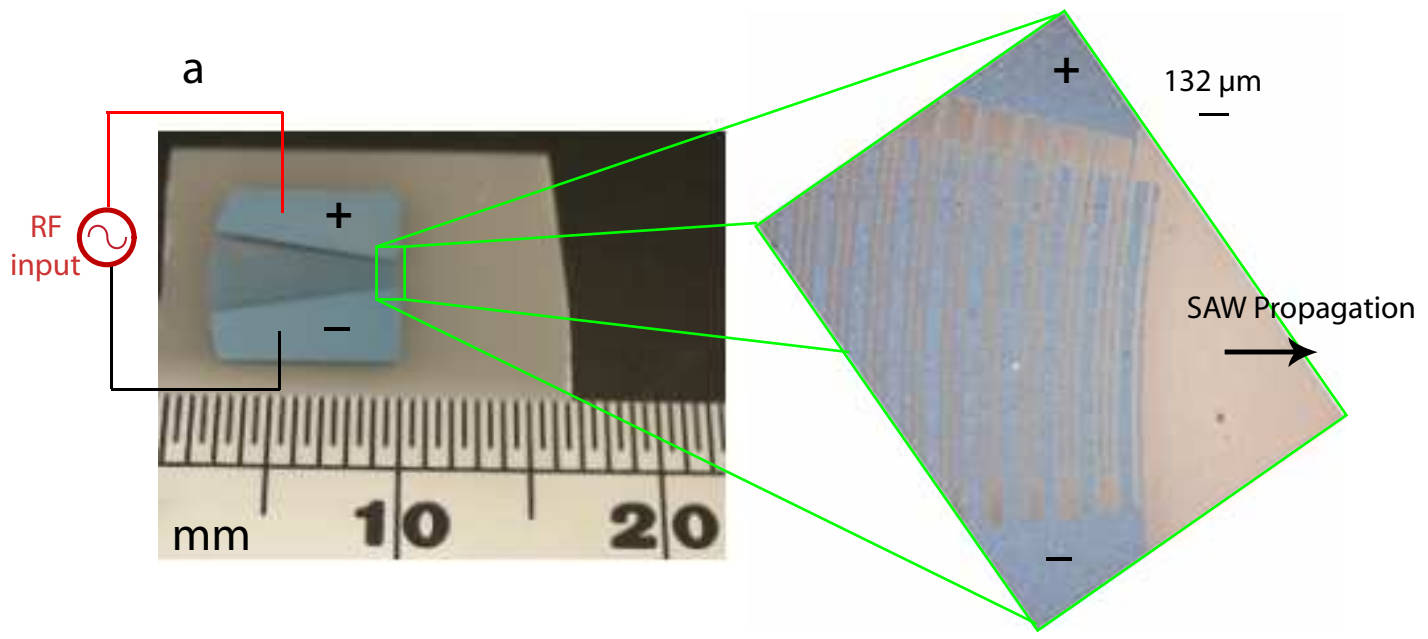

b

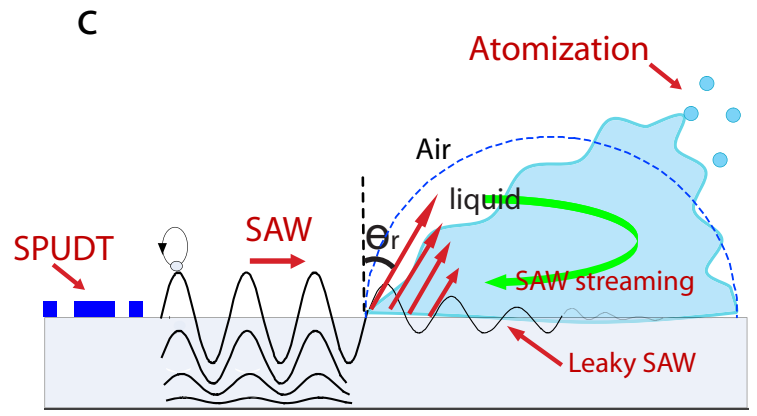

FIG. 1: (a) Image of a $30 \mathrm{MHz}$ SAW atomization device sputtered with SPUDT. (b) Illustration of a pair of SPUDT electrodes. The symbols “+” and "-" stand for the electrodes connected to the positive port and negative port, respectively. In each pair, the negative port has one thin electrode $(1 / 8 \lambda)$ and one wide reflector $(3 / 8 \lambda)$ while the positive port has only one thin electrode $(1 / 8 \lambda)$. The gap between each electrode is $1 / 8 \lambda$. (c) Interaction of the SAW with a liquid drop placed on the substrate. The SAW energy leaks into the drop at the Rayleigh angle and deforms the drop. The vertical component of the SAW destabilizes the capillary waves and eventually results in atomization through break-up of the capillary waves. The horizontal component of the SAW is the source of SAW streaming within the drop.

Protein transport through paper and its subsequent extraction. The model protein solutions employed in our experiment were ovalbumin solutions $(10 \mathrm{mg} / \mathrm{ml}$ and $5 \mathrm{mg} / \mathrm{ml}$ ) and BSA solutions (10 mg/ml and $5 \mathrm{mg} / \mathrm{ml})$. Polyester-cellulose clean room paper strips $(0.5 \mathrm{~cm}$ wide and $2 \mathrm{~cm}$ long) were used. $20 \mu \mathrm{l}$ of protein solution was loaded into each paper strip. Then the paper strips were left at room temperature for drying. By adding a small volume of solvent (water) to wet the 
paper, the protein molecules were extracted out from the paper with the liquid by the SAW. Protein electrophoresis was then employed to examine the properties of the extracted protein molecules (see Sec. III B).

Yeast cell transport through paper and its subsequent extraction. Yeast cells (Instant Dried Yeast, Lowan Whole Foods, Australia) were employed in the experiment. Methylene violet $\left(\mathrm{C}_{22} \mathrm{H}_{23} \mathrm{ClN}_{4}\right.$, Aldrich), which only penetrated dead or non-viable cells, was used to test the viability of the yeast cells $[37,38]$. We cultured the yeast cells $(\sim 20 \mu \mathrm{g} / \mathrm{ml})$ in a sugar-water $(\sim 40 \mu \mathrm{g} / \mathrm{ml})$ solution overnight ( $\sim 12$ hours) at room temperature. In order to eliminate potentially dead or non-viable cells, we selected the cell suspensions from the middle part of the solution in the experiment, avoiding cells floating at the top or cells precipitating at the bottom of the beaker, and confirmed via methylene violet staining that a large majority of these cells were viable. Then, $20 \mu$ cell solution was loaded onto a small piece of polyester-cellulose clean room paper $(0.5 \mathrm{~cm}$ wide and $2 \mathrm{~cm}$ long) for later extraction. The paper was subsequently dried. In our experiments, the dried paper strips were preserved for no more than 3 hours before remoistening using $40 \mu \mathrm{l}$ water for extraction and atomization (see Sec. III C). The cells were collected as atomized onto a clean glass slide used to test the results of the process.

\section{RESULTS AND DISCUSSION}

\section{A. Extraction and atomization of liquid from paper}

The images shown in Fig. 3 obtained with the use of a high speed camera show atomization of water from a thin film that is, over time, pulled out from a meniscus that initially forms at the edge of the paper upon excitation with the SAW. In Fig. 3, frames $a-c$ gradually show the liquid being pulled out from the paper starting upon SAW irradiation. The destabilization of the capillary wave at the meniscus then leads to atomization (frame $c$ ) after about two seconds of SAW excitation. An increase in the power resulted in stronger atomization (frame $d$ ), consuming the liquid much faster than at lower powers, and leading to faster flow rates inside the paper. 


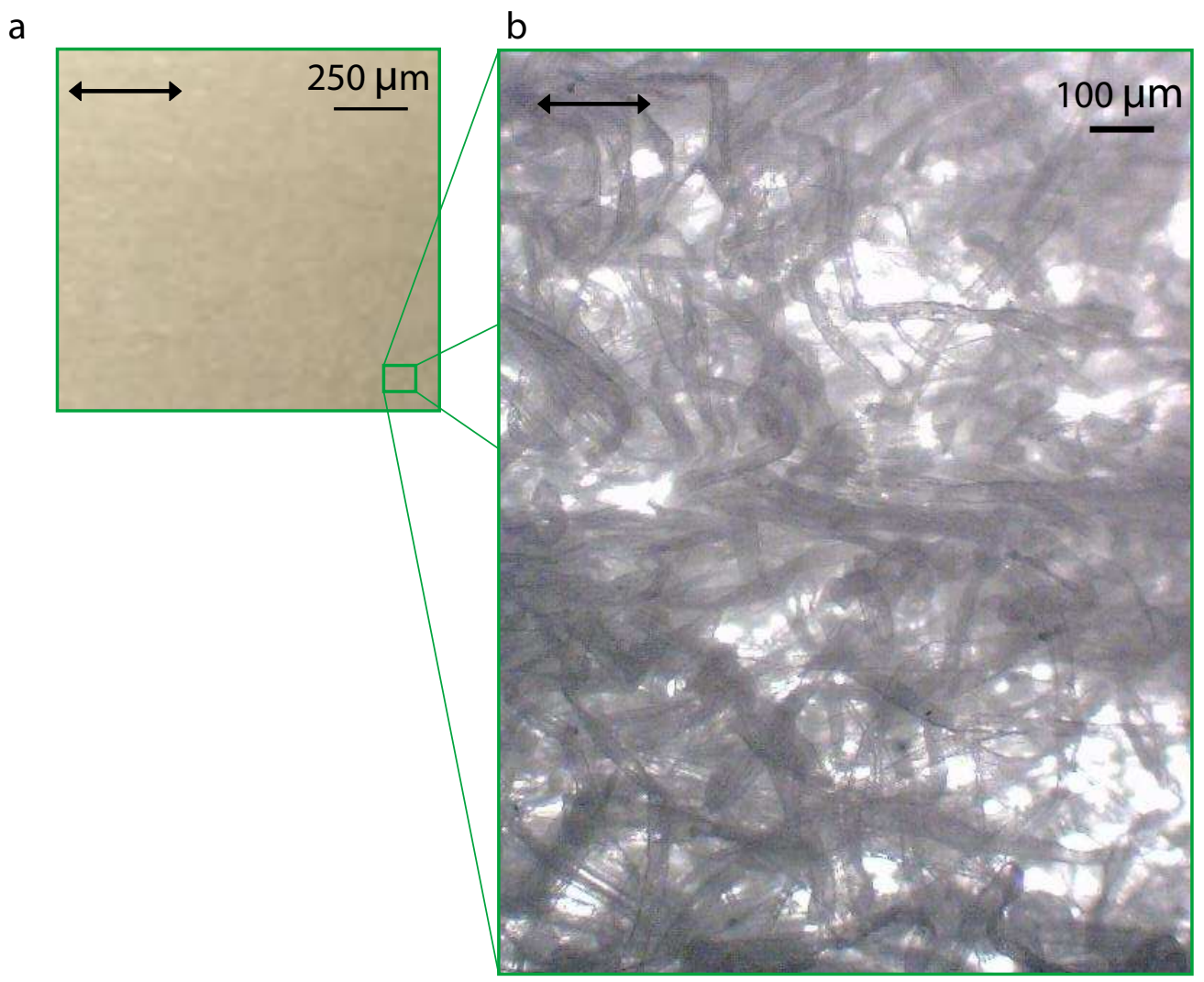

FIG. 2: Images of the polyester-cellulose clean room paper used in our study; (a) shows the polyester-cellulose clean room paper and (b) is a magnified image showing the fibers. The arrows indicate the preferred flow direction defined by the nonuniform fiber orientation and density. The pore size of the paper is on the order of $10-100 \mu \mathrm{m}$ and the width of the fibers are each around $20 \mu \mathrm{m}$.

Upon SAW excitation, Schlichting streaming (or boundary layer streaming) [39, 40], is the dominant mechanism driving fluid motion within the boundary layer adjacent to the substrate through viscous shear, pulling out a thin film of liquid from the contact interface between the wet paper and SAW towards the SAW irradiation. As the fluid film increases in volume and especially depth, long range Eckart streaming appears [39, 40], driving flow in the bulk of the fluid in the direction of the SAW and in opposition to the Schlichting streaming, resulting in the deformation of the free surface perpendicular to the SAW device surface and consequently leading to capillary destabilization and breakup.

This ability to extract liquid out of paper can be useful in various ways, including 
b

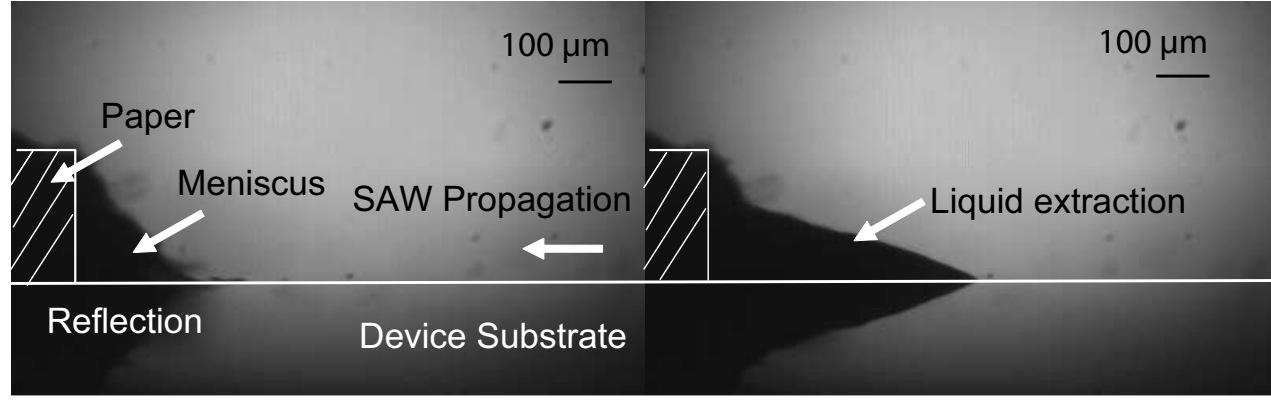

C

d

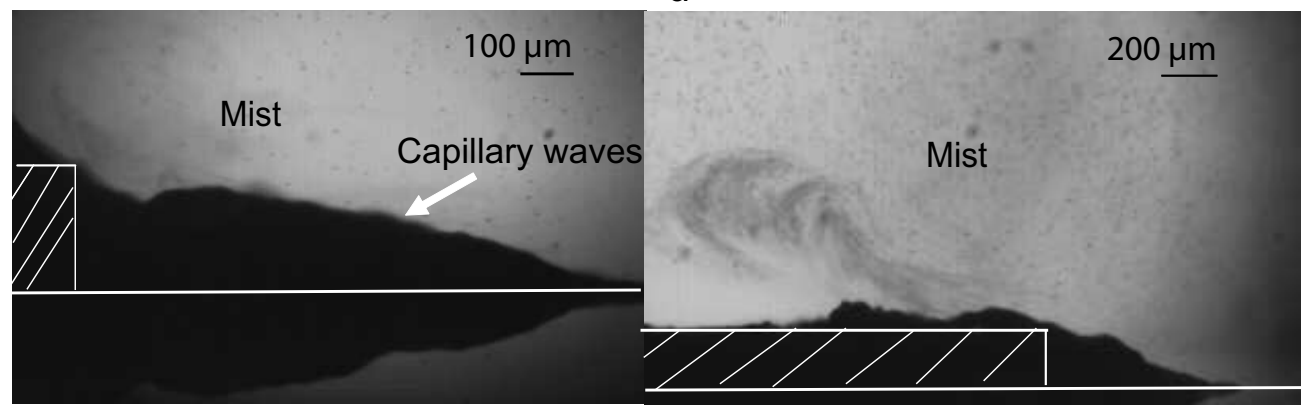

FIG. 3: Atomization of the fluid supplied by paper to the SAW substrate over time. The crosshatched box on the left indicates the position of the paper strip. There is about $98 \mathrm{~ms}$ between frames $a$ and $b$, and about $2 \mathrm{~s}$ between frames $a$ and $c$. Frame $d$ shows stronger atomization as a consequence of a higher input power.

nebulization for pulmonary drug delivery [23]. The most advanced nebulizers on the market are devices employing either a passive vibrating mesh (VM) or a self-vibrating aperture plate (AP) [21]. However, both VM and AP nebulizers require disassembly and careful cleaning after each nebulization to prevent the drug from blocking the apertures and chemicals contained in drug solution from damaging the device. Moreover, the sophistication required in manufacturing the aperture plate increases manufacturing costs. A SAW atomizer with a paper-based fluid supply guide as demonstrated here is promising because of its simplicity and the ease of replacing the paper strip after nebulization. Fig. 4 shows a prototype of the nebulizer utilizing this technology [43]. A small strip of the paper is placed partially inside a capillary tube which is, in turn, connected to a reservoir. Since the paper is so small, its properties, including its porous structure, absorption, and even the effects of evaporation may be ignored. The paper essentially performs as a channel, continuously transporting liquid from the reservoir 


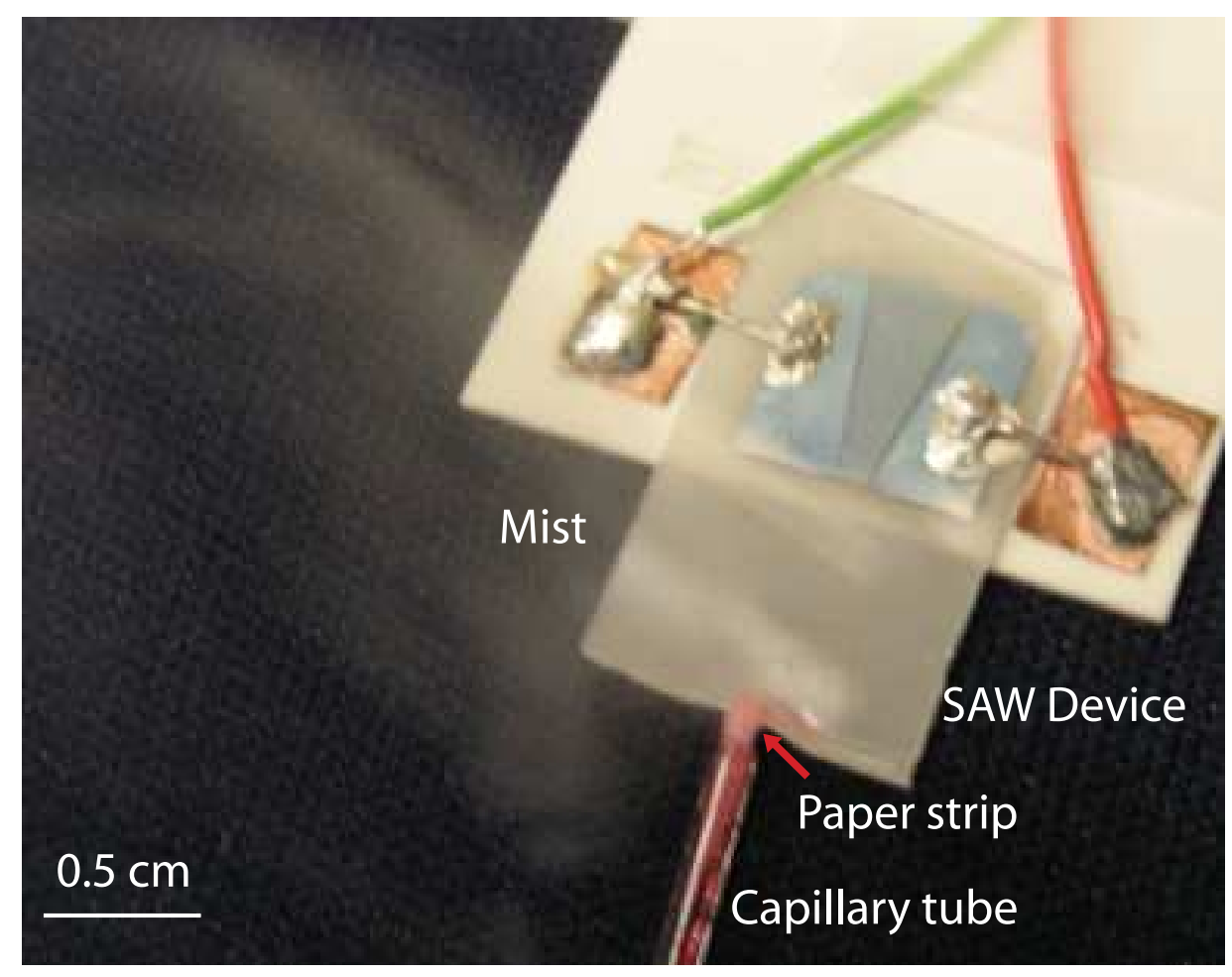

FIG. 4: A prototype of an atomizer utilizing the paper-based liquid transport scheme. A tiny strip of paper is placed into the tip of a capillary tube filled with dye-laden water. Most of the paper is inside the capillary tube, while only the tip (1-2 mm in length) is outside and in contact with the atomizer surface. The outer diameter of the capillary tube is $2 \mathrm{~mm}$ while its inner diameter is about $1 \mathrm{~mm}$. The other end of the capillary tube can be placed into a large reservoir for extended operation; no other pumping is needed as the fluid continuously flows to the atomizer.

through the capillary tube to the atomizer surface.

Beyond liquids, particles preserved inside the paper can also be extracted out of the paper, carried away by the atomized liquid. Fig. 5 shows the water gradually pushing the dye front towards the SPUDT in the polyester-cellulose clean room paper strip $(0.5 \mathrm{~cm}$ wide by $6 \mathrm{~cm}$ long) upon SAW excitation. The paper was first dipped thoroughly in the red dye-laden water. Then the wet red paper was placed upon the SAW device, with one of its end placed at the focus of SAW irradiation provided from the SPUDT. Dye-free (i.e., "clean") distilled water was loaded on the glass substrate at the other end of this paper strip. Allowing the clean water to come into contact with the paper, a thin liquid film (shown in Fig. 5(b) indicated by the arrow) was extracted from the paper towards the SAW irradiation. SAW atomization from the meniscus - the thin film-continuously 

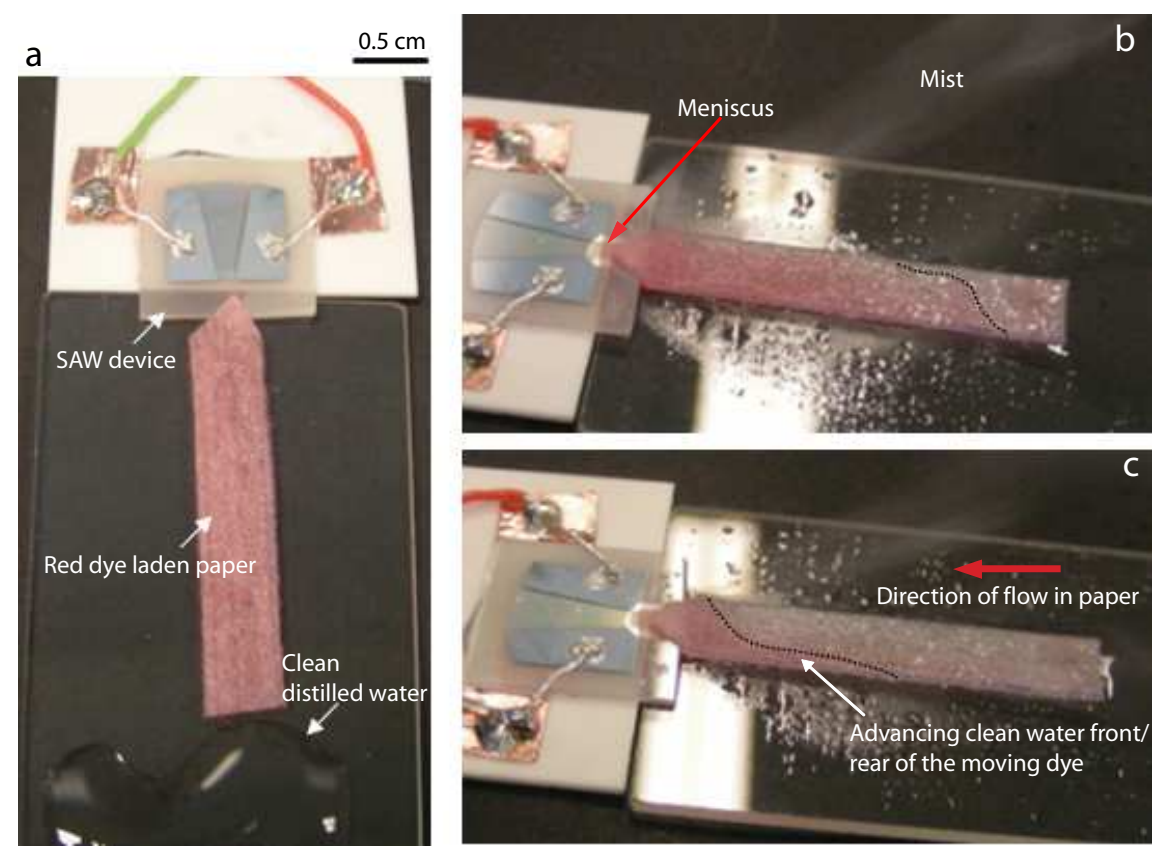

FIG. 5: Image (a) shows the tip of a red dye-laden paper placed on the SAW device before turning on the SAW. Images (b) and (c) gradually show the clean water, which was originally placed at the other end of the paper strip, flowing through the paper, washing off the red dye. The (red) arrow in (b) shows a meniscus, or a thin liquid film, formed at the contacting interface between the wet paper and the SAW, being pulled towards the SAW irradiation due to boundary layer streaming. To aid visualization, the line drawn marks the clean water front, or the rear of the moving dye. Approximately 10 seconds elapsed between images (a), (b) and (c).

pulled the clean water through the paper, subsequently extracting the dye from the paper and atomizing the molecules within the aerosol mist. By replacing the dye with other chemicals or biological molecules, this technique can be used for various applications, as demonstrated in the following sections.

\section{B. Extraction of protein molecules}

Here, we aim to show that chemicals, for example, protein molecules, can be extracted from paper within the extracted liquid. This technology can be used to replace vials with paper to preserve and carry various kinds of chemicals, reagents, proteins, DNAs, and even cells, especially in small quantities; further, as an extension of the paper-based diagnostic device [6-8], we can extract reacted diagnostic protocols out 
of the paper for further examination using this technique.

The thickness of the polyester-cellulose clean room paper strip is about $200 \mu \mathrm{m}$. We prepared the paper strips and preserved the protein (ovalbumin and BSA) molecules as the method described in Sec. II B. When the paper strips were dry, the solvent (DI water) was evaporated and the protein molecules were preserved in the paper. About $40 \mu \mathrm{l}$ water was then loaded to thoroughly wet the paper strip, and the latter was placed upon the SAW device with its tip placed at the focal point of the SAW irradiation. The atomization of the meniscus formed at the edge of the paper acted to continuously pull water from the paper, extracting the protein molecules along with the water out of the paper as an aerosol. The aerosol mist was then collected and tested using an Experion $^{T M}$ Automated Electrophoresis System (Pro260, Bio-Rad laboratories, USA). Figure 6 shows the protein electropherogram obtained by this system. The Experion ${ }^{T M}$ protein fluorescent dye binds to the protein molecules and fluoresces under ultraviolet light, the subsequent fluorescence spectrum being generated by the Experion ${ }^{T M}$ Automated Electrophoresis System software. Smaller molecules travel faster and thus peaks associated with their fluorescence appear earlier than larger molecules. Peaks 2 and 8 are lower $(1.2 \mathrm{kDa})$ and upper $(260 \mathrm{kDa})$ markers, respectively. Peaks 1 and 3 through 5 are the system peaks automatically generated by the system. Peak 6 is the main peak generated by both the ovalbumin and BSA molecules. Peaks generated before the main peak, referring to smaller protein molecules, suggest degradation of these protein molecules. On the other hand, peaks generated after the main peak, corresponding to larger protein molecules, suggest molecular aggregation, though the protein is still considered to be viable. In both Fig. 6(a) and 6(b) there is no obvious peak associated with the extracted-protein samples appearing before the main peak, suggesting that there is no obvious degradation of both ovalbumin and BSA molecules through the extraction and atomization process. Peaks 7 and 8 suggest the existence of some slight aggregation; however, these aggregations already existed in the original sample as a result of high concentrations of protein prior to use. Fig. 7, clearly showing the relative molecule sizes of protein samples, is a virtual gel-electropherogram.

The Data in Table I provide specific results from the experiments: molecular masses, the percentage of degradation compared to the total amount of the test sample, and the output: the percentage of proteins extracted out of the paper compared to the amount 


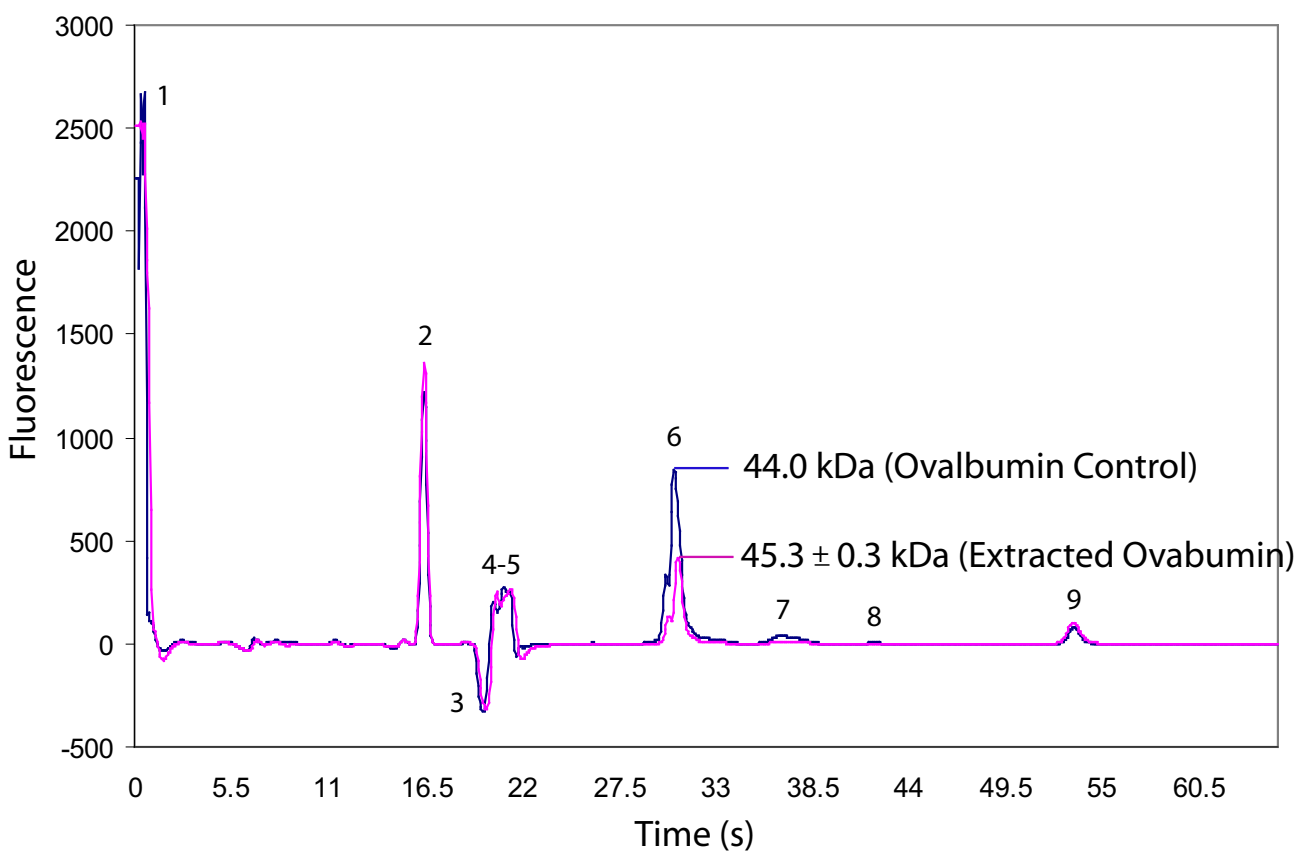

(a) Ovalbumin electropherogram

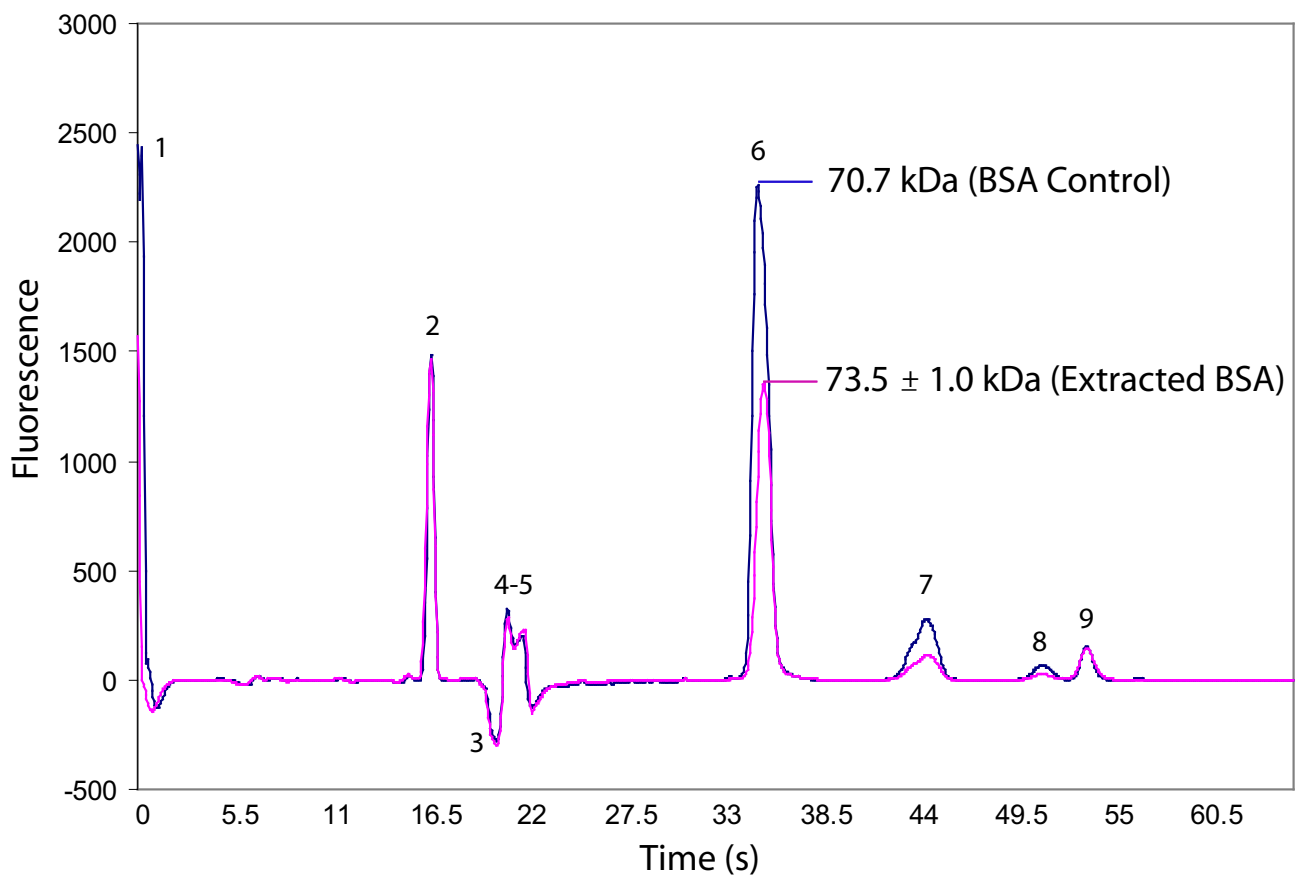

(b) BSA electropherogram

FIG. 6: The protein electropherograms of (a) ovalbumin solutions and (b) BSA solutions. The blue curves represent the original ovalbumin/BSA solution before loading paper-the control, and the red curves represent the averaged fluorescence responses of four ovalbumin and BSA samples after atomization, respectively. 


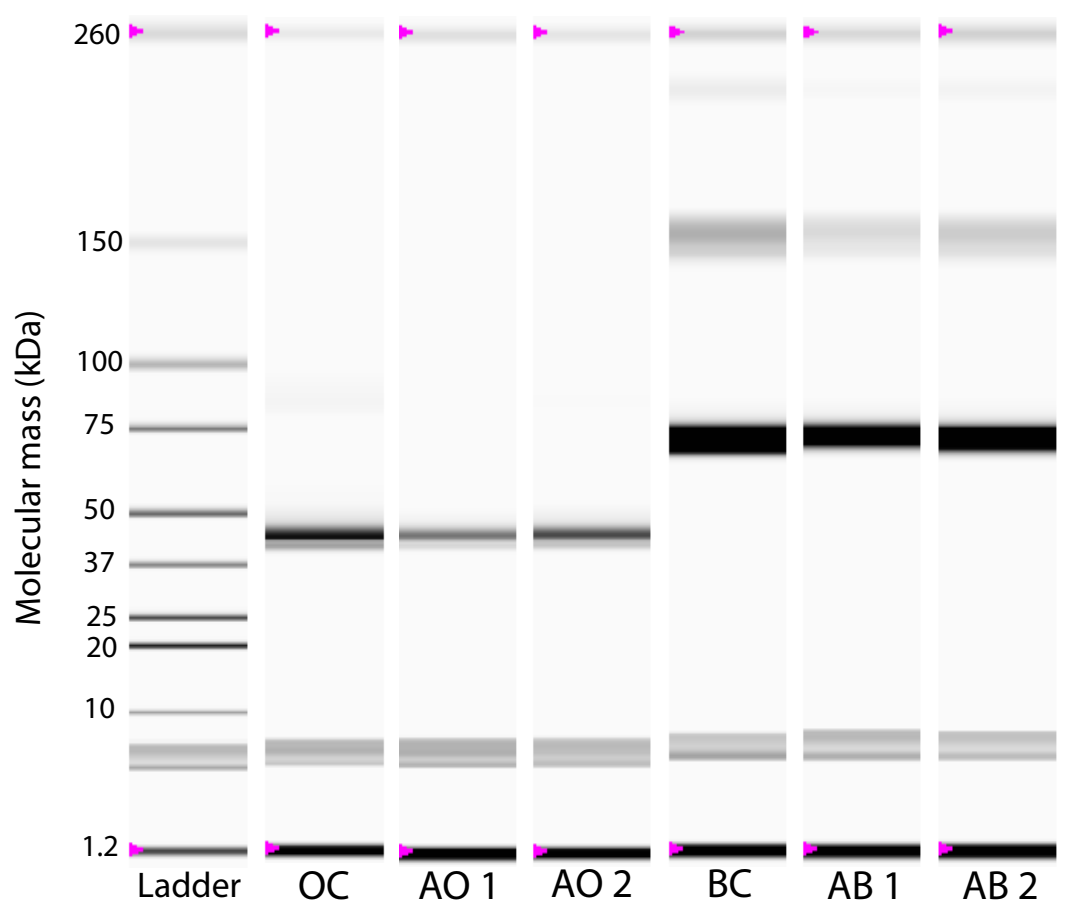

FIG. 7: The gel image of the protein electropherogram. The first column on the left is the ladder associated with molecular masses from $10 \mathrm{kDa}$ to $260 \mathrm{kDa}$. The ovalbumin control (OC) and BSA control (BC) columns stand for the original ovalbumin/BSA solutions before loading onto paper. Ovalbumin samples 1 and 2 (AO 1 and AO 2) and BSA samples 1 and 2 (AB 1 and AB 2) are examples of atomized protein molecules extracted from paper.

TABLE I: Data obtained through protein electrophoresis.

\begin{tabular}{|c|ccc|}
\hline & \multicolumn{4}{|c|}{ Molecular Mass (kDa) } & Degradation (\%) & Output (\%) \\
\hline Ovalbumin Control & 44.0 & 0 & - \\
\hline Atomized ovalbumin & $45.3 \pm 0.3$ & $0.8 \pm 0.4$ & $55.75 \pm 6.8$ \\
\hline BSA Control & 70.7 & 0 & - \\
\hline Atomized BSA & $75.3 \pm 1$ & $0.4 \pm 0.3$ & $67.2 \pm 8.9$ \\
\hline
\end{tabular}

originally loaded into the paper. It is clear that there is almost no degradation $(<1 \%)$ during the extraction-atomization process. Further we are able to extract more than half of the protein molecules loaded into the paper if the amount of water used is sufficient to thoroughly wet the whole paper in order to form a meniscus at the paper's edge. 


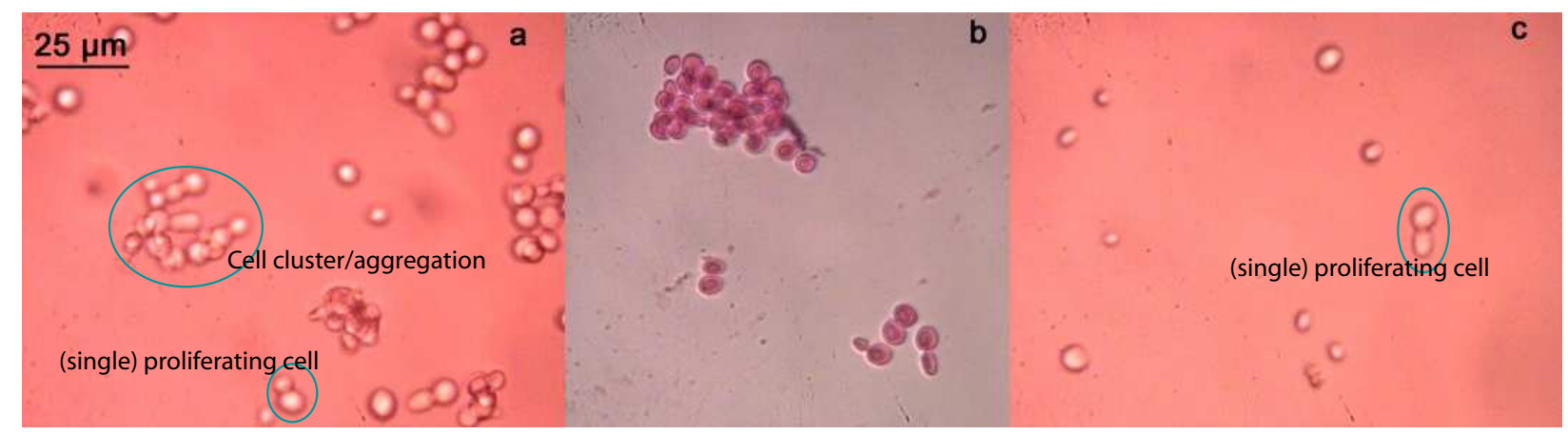

FIG. 8: Yeast cells stained with methylene violet. (a) The original yeast cell sample was directly taken from the solution culture. These viable cells are not stained by methylene violet. In this image, proliferating cells and cell clusters (aggregations) are easy to see. (b) The original sample was treated with $90 \%$ ethanol, killing the cells; the cells now easily stain with methylene violet. (c) The cells preserved in paper were subsequently extracted out of the paper within the atomization mist. Methylene violet does not stain these cells, suggesting they remain viable. A proliferating cell is also shown in this image as circled.

\section{Extraction of yeast cells}

It has already been proven by Li, et al., that at low power levels ( $<120 \mathrm{~mW})$, SAW streaming does not affect the viability of cells [37, 41]; however, at high powers, whether the SAW atomization can affect the viability of yeast cells has yet to be investigated. Furthermore, cell preservation and extraction using SAW, if successful, can easily replace the use of vials for transporting cells, or other bioparticles. Therefore, as an initial test of the technique, it is expedient to determine whether cells can survive this in-paper extraction and atomization process using SAW. Fig. 8 shows the yeast cells survived the SAW extraction-atomization process. The power was set to $2 \mathrm{~W}$, and the whole extraction-atomization process only took about 10-15 seconds. When cells were extracted through paper, they passed through the porous structure created by the overlapping paper fibers, which resemble internal microchannels. From Fig. 2, we can see that the smallest pore size, the gap between those overlapping fibers, is less than $10 \mu \mathrm{m}$ (see Fig. 2). Therefore, when the cell suspensions were traveling through, the large particles, more precisely, the cell clusters or aggregations $(\geq 20 \mu \mathrm{m})$, were jammed between the fibers, while single cells and single proliferating cells $(\sim 5 \mu \mathrm{m})$, have a greater prob- 
ability of passing through the pores of the paper with the extraction fluid, suggesting a means for rapid cell filtration or separation.

\section{CONCLUSIONS}

Lab-in-paper microfluidics is a promising approach that addresses many of the shortcomings of traditional microfluidics technology. We provide a technique for enhancing the usefulness of apper microfluidics with a method of extracting liquid out of the paper. Though past studies have considered what can be done in the paper, for example, to transport and analyze chemical protocols inside the paper by means of color indicators $[8,42]$, one is unable to extract the results out of the paper to analyze them with more sophisticated techniques. SAW atomization is a specific example of how this problem may be solved, acting as an interface between microfluidic devices and mass spectrometry, for example. When a wet paper strip is placed upon the atomizer surface, atomization takes place from a meniscus that forms along an area near the edge of the paper as it is irradiated by SAW. Ovalbumin and BSA were employed as model protein molecules, and were preserved in paper, extracted out by rewetting and SAW irradiation and were found to be in the atomized aerosol. According to protein electrophoresis, there was less than $1 \%$ degradation of either protein molecule, showing the promise of this approach. Successful preservation and extraction of yeast cells in paper also suggests a broader potential application for this technology.

\section{ACKNOWLEDGEMENTS}

Funding for this work was provided through Australian Research Council Discovery Project Grants DP0677321, DP0773221 and DP1092955 and Nanotechnology Victoria Grant BN-004.

[1] J. L. Gay-Lussac, Annales des Chimie et des Physique, 1828, 39, 352-374.

[2] R. Block, E. Durrum and G. Zweig, Soil Science, 1955, 79, 409.

[3] J. Ehrenkranz, Epidemiology, 2002, 13, S15. 
[4] R. C. Willis, Analytical Chemistry, 2006, 78, 5261-5265.

[5] C. D. Chin, V. Linder and S. K. Sia, Lab on a Chip, 2007, 7, 41-57.

[6] A. W. Martinez, S. T. Phillips, B. J. Wiley, M. Gupta and G. M. Whitesides, Lab on a Chip, 2008, 8, 2146-2150.

[7] A. W. Martinez, S. T. Phillips and G. M. Whitesides, Proceedings of the National Academy of Sciences, 2008, 105, year.

[8] A. W. Martinez, S. T. Phillips, E. Carrilho, S. W. T. III, H. Sindi and G. M. Whitesides, Analytical Chemistry, 2008, 80, 3699-3707.

[9] K. Kulkarni, J. Friend, L. Yeo and P. Perlmutter, Lab on a Chip, 2009, 9, 754.

[10] J. McLean, R. Huff and A. Montaser, Applied Spectroscopy, 1999, 53, 1331-1340.

[11] O. A. Basaran, AIChE Journal, 2002, 48, 1842.

[12] L. Y. Yeo, Z. Gagnon and H.-C. Chang, Biomaterials, 2005, 26, 6122-6128.

[13] R. Williams, Drug Development and Industrial Pharmacy, September 2008, 34, 913-922.

[14] M. Gumbleton and G. Taylor, Advanced Drug Delivery Reviews, 2006, 58, 993-995.

[15] G. Firanescu, D. Hermsdorf, R. Ueberschaer and R. Signorell, Physical Chemistry Chemical Physics, 2006, 8, 4149-4165.

[16] K. Powell, Nat Biotechnol, 2004, 22, 1195-1196.

[17] J. Kling, Nature Biotechnology, 2008, 26, 479-480.

[18] A. M. Gañán-Calvo, Physical Review Letters, 1998, 80, 285.

[19] J. C. Ijsebaert, K. B. Gerse, J. C. M. Marijnissen, J.-W. J. Lamers and P. Zanen, Journal of Applied Physiology, 2001, 91, 2735-2741.

[20] R. Lang, The Journal of the Acoustical Society of America, 1962, 34, 6.

[21] J. C. Waldrep and R. Dhand, Current Drug Delivery, 2008, 5, 114-119.

[22] A. Qi, L. Yeo and J. Friend, Physics of Fluids, 2008, 20, 074103.

[23] A. Qi, J. R. Friend and L. Y. Yeo, Lab on a Chip, 2009, 9, $2184-2193$.

[24] L. Y. Yeo and J. R. Friend, Biomicrofluidics, 2009, 3, 012002.

[25] J. Friend, L. Yeo, D. Arifin and A. Mechler, Nanotechnology, 2008, 19, 145301.

[26] M. Alvarez, J. R. Friend and L. Y. Yeo, Nanotechnology, 2008, 19, 455103.

[27] M. Alvarez, L. Yeo and J. Friend, Biomicrofluidics, 2009, 3, 014102.

[28] M. K. Tan, J. Friend and L. Yeo, Lab on a Chip, 2007, 7, 618-625.

[29] M. Alvarez, J. Friend and L. Yeo, Langmuir, 2008, 24, 10629-10632. 
[30] S. Girardo, M. Cecchini, F. Beltram and R. Cingolani, Lab on a Chip, 2008.

[31] M. Cecchini, S. Girardo, D. Pisignano and R. Cingolani, Applied Physics Letters, 2008.

[32] H. Li, J. R. Friend and L. Y. Yeo, Biomaterials, 2007, 28, 4098-4104.

[33] M. Kurosawa, T. Watanabe and T. Higuchi, Micro Electro Mechanical Systems, MEMS '95, Proceedings. IEEE, 1995.

[34] K. Chono, N. Shimizu, Y. Matsui, J. Kondoh and S. Shiokawa, Japanese Journal of Applied Physics, 2004, 43, 2987-2991.

[35] M. Takeuchi and K. Yamanouchi, IEEE Transactions on ultrasonics. ferroelectrics, and frequency control, 1993, 40, 648-658.

[36] A. Wixforth, C. Strobl, C. Gauer, A. Toegl, J. Scriba and Z. v. Guttenberg, Analytical and Bioanalytical Chemistry, 2004, 379, 982-991.

[37] H. Li, J. R. Friend and L. Y. Yeo, Biomedical Microdevices, 2007, 9, 647-656.

[38] K. A. Smart, K. M. Chambers, I. Lambert and C. Jenkins, Journal of American Society of Brewing Chemists, 1999, 57(1), 18-23.

[39] S. Hardt and F. Schönfeld, Microfluidic Technologies for Miniaturized Analysis Systems, Springer, 2007, p. 364.

[40] Physical acoustics, ed. W. P. Mason, Academic Press, 1965.

[41] H. Li, A. Dasvarma, L. Yeo, J. Friend and K. Traianedes, Biomicrofluidics, 2009, 3, 034102.

[42] X. Li, J. Tian, T. Nguyen and W. Shen, Analytical Chemistry, 2008, 80, 9131-9134.

[43] Please see the associated video showing the atomisation process as Electronic Supplementary Information. 